\title{
Observations of poleward-propagating large-scale traveling ionospheric disturbances in southern China
}

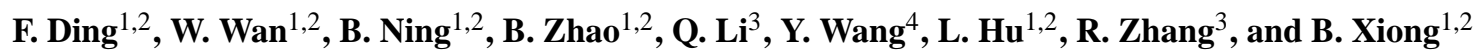 \\ ${ }^{1}$ CAS Key Laboratory of Ionospheric Environment, Institute of Geology and Geophysics, Chinese Academy of Sciences, \\ Beijing, China \\ ${ }^{2}$ Beijing National Observatory of Space Environment, Institute of Geology and Geophysics, Chinese Academy of Sciences, \\ Beijing, China \\ ${ }^{3}$ National Earthquake Infrastructure Service, Beijing, China \\ ${ }^{4}$ National Center for Space Weather, China Meteorological Administration, Beijing, China
}

Correspondence to: F. Ding (dingf@mail.iggcas.ac.cn)

Received: 1 November 2012 - Revised: 5 February 2013 - Accepted: 13 February 2013 - Published: 28 February 2013

\begin{abstract}
We report here on two cases of polewardpropagating large-scale traveling ionospheric disturbances (LSTIDs) in China during a medium-scale storm between 27 May and 1 June 2011. The observations were conducted by making use of the Global Positioning System network and ionosondes in China and Southeast Asia. One northeastward-propagating LSTID occurred on the morning of 30 May, while the other was observed during the nighttime of 1 June. Both poleward-traveling LSTIDs occurred during the storm's recovery phase in southern China's lowlatitude region (geomagnetic latitude $\sim 7.3-24^{\circ} \mathrm{N}$ ) and experienced severe dissipation during their propagation from south to north. Although the initial relative amplitude of the nighttime LSTID was $\sim 60 \%$ larger than that of the morning event, the nighttime event dissipated more quickly than the morning event because of a strong nighttime enhancement in background total electronic content (TEC) during storm time, which led to strong ion-drag dissipation during the evening. The poleward-propagating LSTIDs exhibit a narrower latitudinal range, a smaller amplitude, and a slightly higher elevation compared with the equatorwardmoving LSTIDs observed in the same region. Given these features, the poleward-propagating LSTIDs were likely excited by some local source near southern China. Excitation of secondary LSTIDs during the dissipation of some primary medium-scale disturbances from the lower atmosphere is a possible mechanism.
\end{abstract}

Keywords. Ionosphere (Ionospheric disturbances)

\section{Introduction}

The propagation direction of large-scale traveling ionospheric disturbances (LSTIDs) is closely related to the location of the source and the relative position of the observer with respect to the source. Auroral or sub-auroral activities, such as enhancement of auroral electrojets or particle precipitation, are the primary sources of LSTIDs (Hunsucker, 1982). LSTIDs originating from auroral sources usually propagate southwestward in high-latitude regions (Ding et al., 2007, 2008), and westward deviations from the south seem to be caused by the westward movement of the source region (Hocke and Schlegel, 1996). Song et al. (2012) found that westward deviations from south of LSTIDs increased as the LSTIDs propagated from high to middle latitudes due to the Coriolis force. At middle and low latitudes, the Japanese GEONET observation network has found eastwardpropagating in addition to westward-propagating traveling ionospheric disturbances (TIDs) (Tsugawa et al., 2004). This was assumed to have been caused by the eastward declination of the geomagnetic field.

LSTIDs propagating poleward are rarely observed. Models predict that LSTIDs excited in auroral regions during geomagnetically active periods can propagate across the equator to the opposite hemisphere and travel poleward (Lei et al., 2008; Vadas and Liu, 2009). Poleward-propagating LSTIDs have been observed by several authors, mostly in middleand low-latitude regions (Lei et al., 2008; Bruinsma and Forbes, 2009; Zhou et al., 2012). Based on neutral-density 


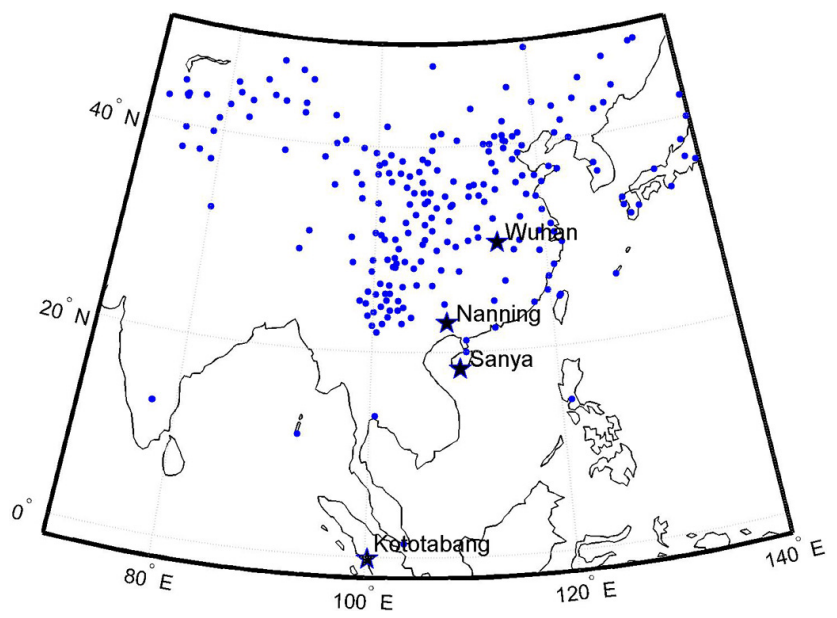

Fig. 1. Location of GPS stations (blue dots) and ionosondes (asterisks) in East Asia.

data provided by CHAMP observations, Bruinsma and Forbes (2009) identified 21 cross-equator LSTIDs during the period 2001-2007, which were excited at high latitudes. The average horizontal scale of these poleward-propagating LSTIDs is $\sim 4800 \mathrm{~km}$. Observations obtained with the highfrequency multistatic backscatter sounding radars at Wuhan $\left(30^{\circ} \mathrm{N}, 114^{\circ} \mathrm{E}\right)$ revealed a poleward-propagating LSTID during a geomagnetically quiet time, which was assumed to be a secondary LSTID that originated from the dissipation of medium-scale gravity waves in the lower atmosphere.

This paper reports on two cases of poleward-propagating LSTIDs at low latitudes during a medium-scale magnetic storm from 27 May to 1 June 2011. The observations were made using TEC data from 241 Global Positioning System (GPS) receivers around China, as well as from ionosondes in this region.

\section{Data and method}

In this study, we collected TEC data from 241 GPS receivers located around China, whereas 188 were from the Crustal Movement Observation Network of China (CMONOC) and 53 from the International GNSS Service (IGS). Figure 1 plots the location of the GPS receivers. The CMONOC is a largescale network aimed at obtaining crustal-movement observations of both high accuracy and high spatial and temporal resolution, using Global Navigation Satellite System (GNSS) as its main observation technique, combined with other spacebased techniques, such as very long baseline interferometry, satellite laser ranging, precise leveling, and precise gravimetry. The network was completed in late 2010 and offered continuous TEC observations since that time.

Using these TEC observations, we constructed twodimensional (2-D) deviation-of-TEC (DTEC) maps to search for LSTIDs over China. The method of map construction and
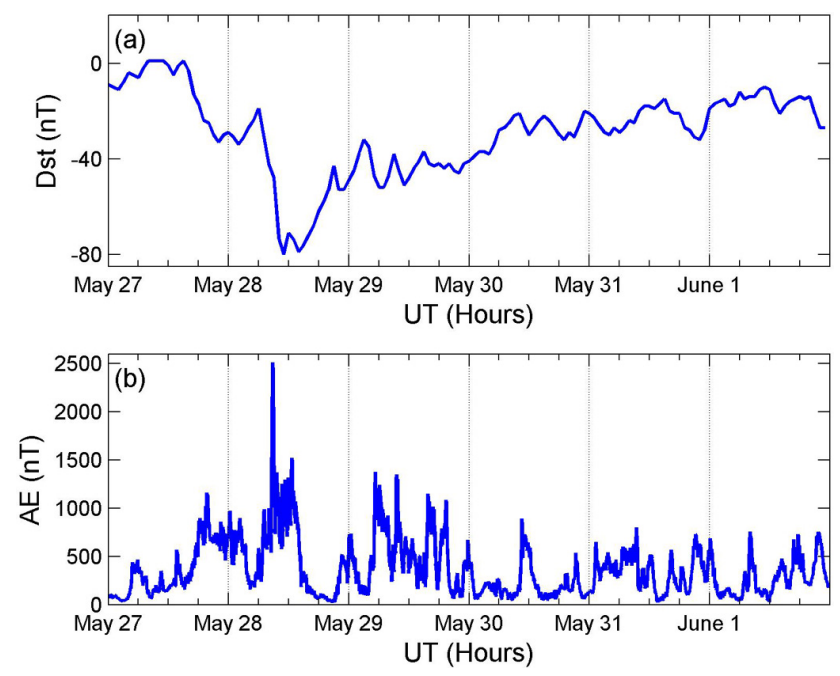

Fig. 2. Temporal variations in the Dst index (a) and AE indices (b) during 27 May-1 June 2011.

determination of LSTID events was introduced in detail by Ding et al. (2012). First, we obtained the TEC perturbation time series by filtering out the background trend from the original TEC series. This background trend is expressed as a linear function of local time, as well as a second-order function of latitude. Next, we divided the area of interest (15$55^{\circ} \mathrm{N}, 70-140^{\circ} \mathrm{E}$ ) into pixels with a size of $1^{\circ}$ latitude $\times 1^{\circ}$ longitude. We calculated the amplitude of the TEC perturbation in each pixel by taking the average of the TEC variations for all GPS-satellite receiver paths whose ionospheric pierce points crossed the pixel during the time of the observation. Thus, we obtained the 2-D TEC variation map for the relevant time frame. We repeated this calculation to obtain a sequence of maps with a temporal resolution of $5 \mathrm{~min}$. If LSTIDs were passing by, there would be regularly moving band-like structures in the data. On the basis of these maps, we calculated the propagation parameters such as the periods, phase velocities, azimuth angles, and amplitudes.

We also used ionogram data from four low-latitude ionosonde stations at Wuhan $\left(30.5^{\circ} \mathrm{N}, 114.4^{\circ} \mathrm{E}\right)$, Nanning $\left(22.8^{\circ} \mathrm{N}, 108.3^{\circ} \mathrm{E}\right)$, Sanya $\left(18.3^{\circ} \mathrm{N}, 109.6^{\circ} \mathrm{E}\right)$, and an equatorial station at Kototabang $\left(0.2^{\circ} \mathrm{S}, 100.3^{\circ} \mathrm{E}\right)$ (Fig. 1). The stations at Wuhan and Sanya belong to the Beijing National Observatory of the Space Environment, the station at Nanning belongs to the National Center for Space Weather of the China Meteorological Administration, and the data at Kototabang are from the World Data Center (WDC) for the Ionosphere at Tokyo's National Institute of Information and Communications Technology.

\section{Observations}

We conducted the observations during a medium-scale storm from 27 May to 1 June 2011. Figure 2 draws the temporal 
(a) UT=01:40

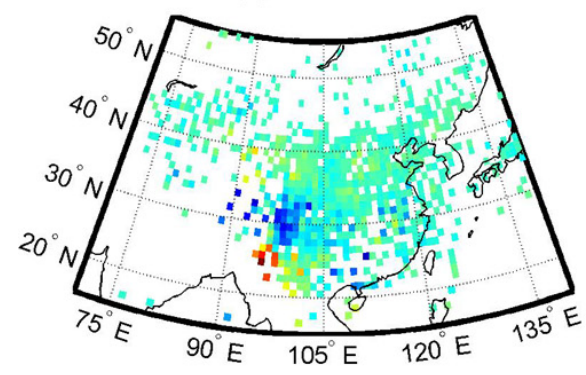

(c) UT=02:20

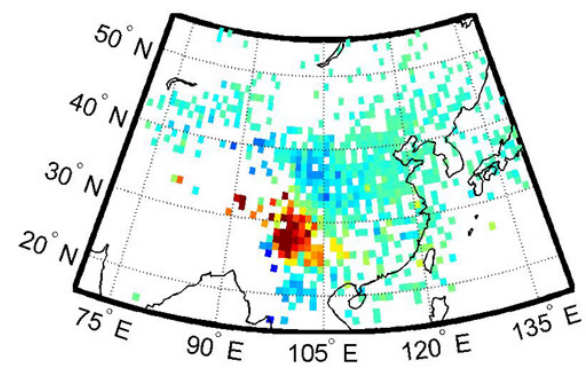

(e) UT $=03: 00$

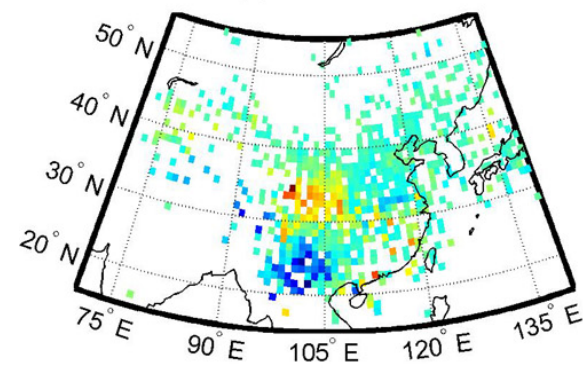

(g) UT=03:40

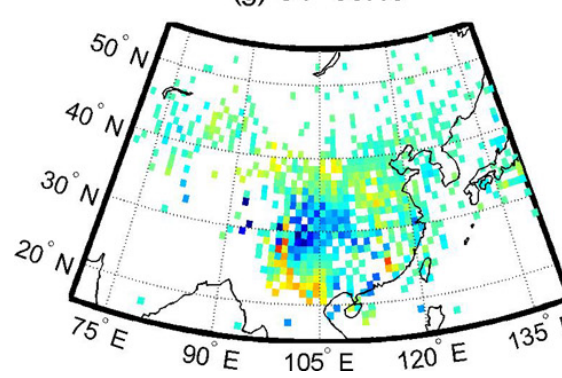

(b) UT=02:00

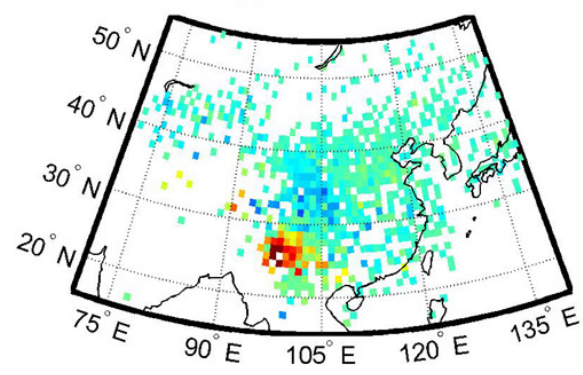

(d) UT $=02: 40$

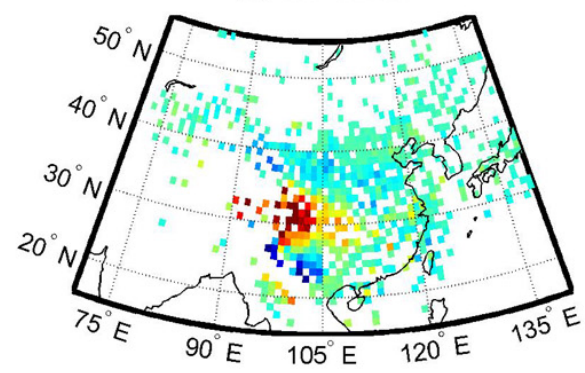

(f) $U T=03: 20$
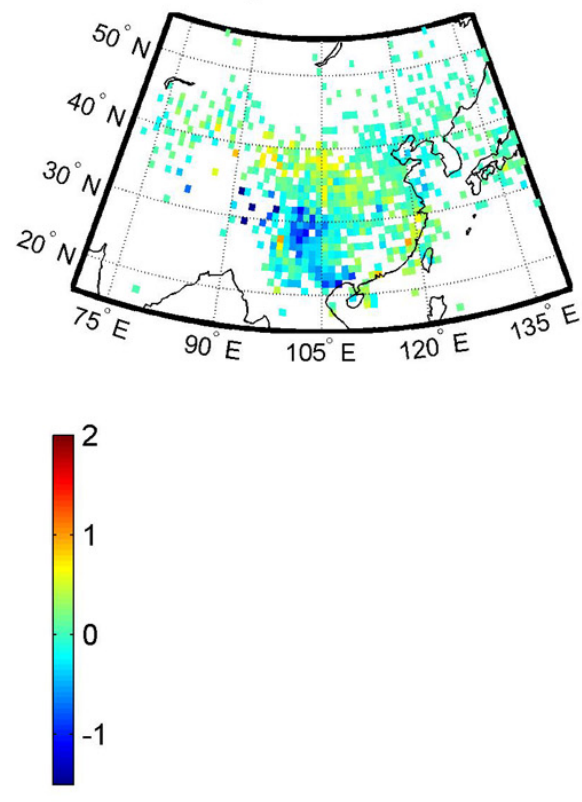

Fig. 3. Sequence of 2-D TEC maps over China between 01:40 and 03:40 UT (i.e., 09:40-11:40 LT) on 30 May 2011. The color interval represents the deviation of TEC (units: TECU).

variation of the Dst index (a) and AE index (b) during this period. The storm began at $\sim$ 15:00 UT on 27 May. The Dst dropped to a minimum of $-80 \mathrm{nT}$ on 28 May, following the onset of a substorm, with an increase of the AE index of up to $-2500 \mathrm{nT}$. The storm went through its recovery phase during the next four days, which was accompanied by some slight increase in the AE index of less than $1000 \mathrm{nT}$. Detailed storm information has been reported by Ding et al. (2012).
We observed two poleward-propagating LSTID events during this period. Both were observed during the storm's recovery phase, on 30 May and 1 June, respectively. The first event occurred in the morning at 01:40-03:40 UT (i.e., at a local time, LT, of 09:40-11:40 LT) on 30 May. Figure 3 shows the 2-D plots of the DTEC during this period. Band-like structures can be seen to move from the southwest to the northeast over a distance of $\sim 2500 \mathrm{~km}$. The amplitude of the disturbance ranges from -1.4 to $1.0 \mathrm{TECU}$ 
(a) UT=12:40

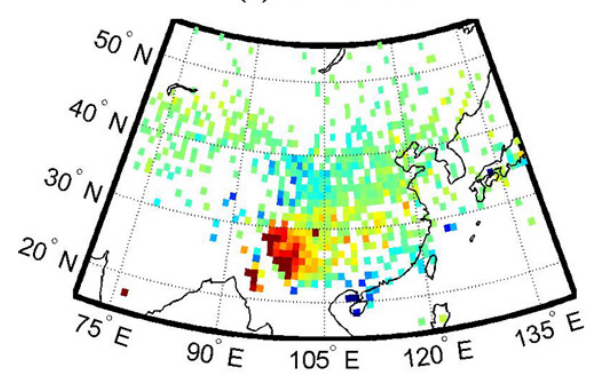

(c) UT $=13: 20$

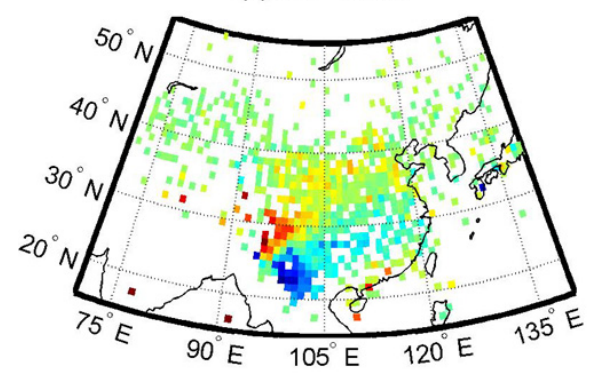

(e) UT $=14: 00$

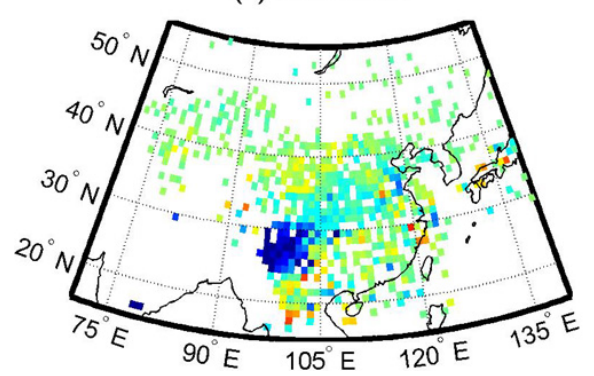

Fig. 4. As for Fig. 3, but for 12:40-14:00 UT (i.e., 20:40-22:00 LT).

$\left(1 \mathrm{TECU}=10^{16}\right.$ electron $\left.\mathrm{m}^{-2}\right)$. Based on the movements of the phase fronts, the horizontal velocity and propagation azimuth (east of north) were measured at $410 \pm 66 \mathrm{~m} \mathrm{~s}^{-1}$ and $21 \pm 6^{\circ}$, respectively. The second event was observed during 12:40-14:00 UT (20:40-22:00 LT), before midnight on 1 June (see Fig. 4). This LSTID propagated northwestward over a distance of $\sim 3000 \mathrm{~km}$, with an amplitude range of \pm 2 TECU. The horizontal velocity and propagation azimuth of the second LSTID were $319 \pm 52 \mathrm{~m} \mathrm{~s}^{-1}$ and $333 \pm 9^{\circ}$, respectively.

Figure 5 presents the TEC variation as a function of horizontal distance and universal time, during the periods when the LSTIDs occurred on the morning of 30 May (Fig. 5a) and the evening of 1 June (Fig. 5b). The horizontal distance is calculated along the one-dimensional axis perpendicular to the wavefronts. The origins of the axis were set at $\left(20^{\circ} \mathrm{N}, 98^{\circ} \mathrm{E}\right)$ for the morning event and $\left(20^{\circ} \mathrm{N}, 105^{\circ} \mathrm{E}\right)$ for the nighttime event; i.e., at the positions where the band-like structures that were associated with the LSTIDs first appeared. Again, we see clearly two LSTIDs in Fig. 5, shown as oscillations in the (b) UT=13:00

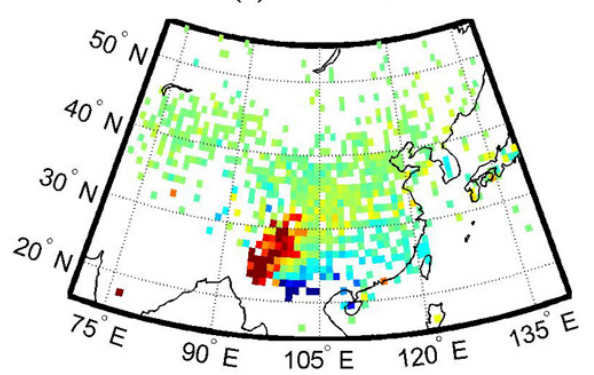

(d) UT=13:40
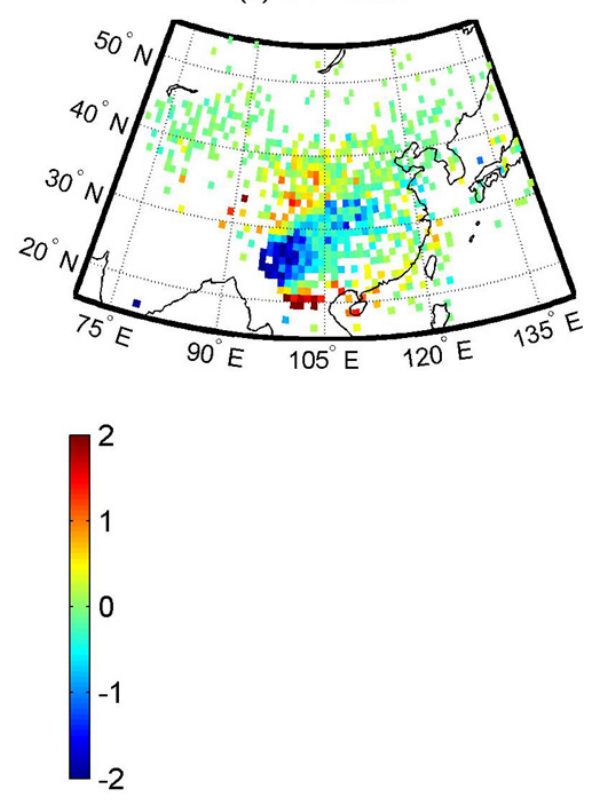

DTEC as a function of time and propagation distance. The horizontal range of both LSTIDs exceeds $2000 \mathrm{~km}$, and the horizontal range shown in the figure is $400-500 \mathrm{~km}$ longer than the distance shown in the 2-D plots (see Figs. 2 and 3). This difference may be caused by the relatively sparse distribution of GPS receivers in northern China, which makes it difficult to obtain enough data to construct 2-D perturbation maps. Figure 5 also shows that the live duration of these LSTIDs is much longer than that of the LSTIDs shown in Figs. 2 and 3. For example, while the LSTID on 30 May shows a clear phase front for a duration of $2 \mathrm{~h}$ (see Fig. 3), Fig. 5a indicates a duration of $\sim 7 \mathrm{~h}$. We estimated from Fig. 5 that the periods of the LSTIDs are $1.81 \pm 0.15$ and $1.84 \pm 0.14 \mathrm{~h}$.

Note that for both events the absolute amplitude decreased rapidly during their propagation. For example, on 30 May, the positive amplitude decreased from $\sim 0.7$ TECU at a horizontal distance of $0 \mathrm{~km}$ at 01:40 UT, to $\sim 0.3 \mathrm{TECU}$ at $2500 \mathrm{~km}$ at 02:30 UT (see Fig. 5a). A similar decrease in amplitude also occurred for the LSTID on 1 June (see Fig. 5b). 

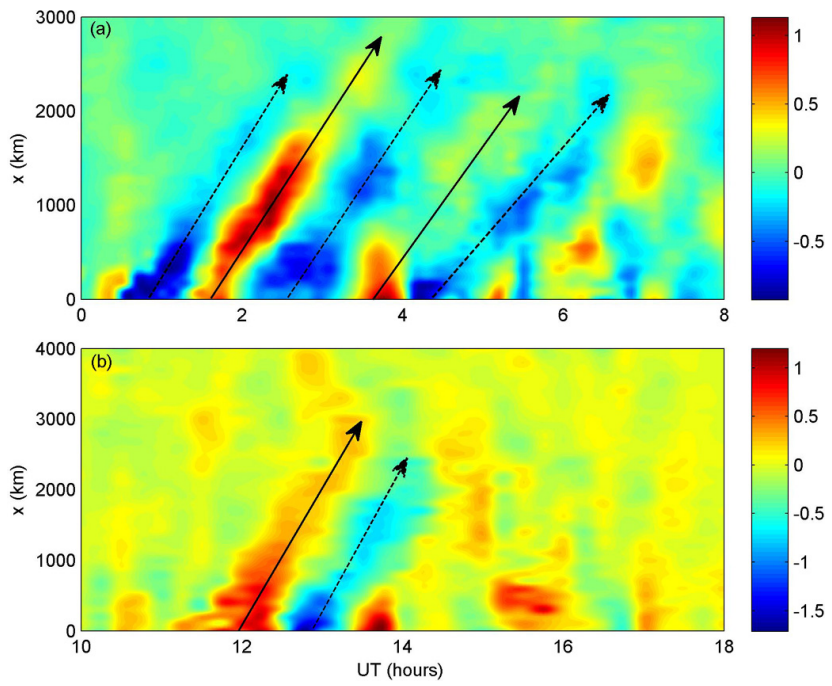

Fig. 5. TEC variation as a function of horizontal distance and universal time (UT) during the periods when the LSTIDs occurred on the morning of 30 May (panel a) and the evening of 1 June (panel b). The horizontal distance is calculated along the one-dimensional axis, $x$, perpendicular to the wavefronts and directed toward an azimuth of $21^{\circ}$ for the morning LSTID on 30 May and toward $333^{\circ}$ for the nighttime event on 1 June. The origins of the axis were set at $\left(20^{\circ} \mathrm{N}, 98^{\circ} \mathrm{E}\right)$ for the morning event and $\left(20^{\circ} \mathrm{N}, 105^{\circ} \mathrm{E}\right)$ for the nighttime event, the positions where the band-like structures that are associated with the LSTIDs first appeared. The black lines mark the phase fronts in the $\mathrm{x}-\mathrm{UT}$ plane.

Such decreases could be related to dissipation or to the latitudinal change in the background TEC. To estimate the dissipation of the LSTIDs, we calculated their relative amplitudes by dividing the absolute amplitudes by the background TEC, which was obtained from Global Ionosphere Maps (GIM) data from the Jet Propulsion Laboratory (JPL) data.

Figure $6 \mathrm{a}$ and $\mathrm{b}$ show temporal variations in relative amplitude at fixed horizontal locations, $x$, ranging from 0 to $2500 \mathrm{~km}$ at intervals of $500 \mathrm{~km}$. Both LSTID events dissipated during their propagation from south to north, but the nighttime event dissipated more quickly than the morning event. At $x=0 \mathrm{~km}$, the morning and nighttime events exhibit a maximum amplitude of $3 \%$ (Fig. 6a) and $5 \%$ (Fig. 6b), respectively. The amplitude of the morning event decreased from $3 \%$ at $x=0 \mathrm{~km}$ to $0.8 \%$ at $x=2000 \mathrm{~km}$, when it increased slightly again and reached $1 \%$ at $x=2500 \mathrm{~km}$. For the nighttime event, the amplitude decreased continuously from $5 \%$ at $0 \mathrm{~km}$ to $1 \%$ at $2500 \mathrm{~km}$. Although the amplitude of the nighttime LSTID was $\sim 60 \%$ larger than that of the morning event at $x=0$, the amplitudes of both events decrease to $1 \%$ after they traveled a distance of $2500 \mathrm{~km}$.

It is most likely that the difference in dissipation of the two LSTID events is caused by storm-time variations in the background TEC. Using JPL GIM data, we show in Fig. 6c the latitudinal variations in the background TEC along the
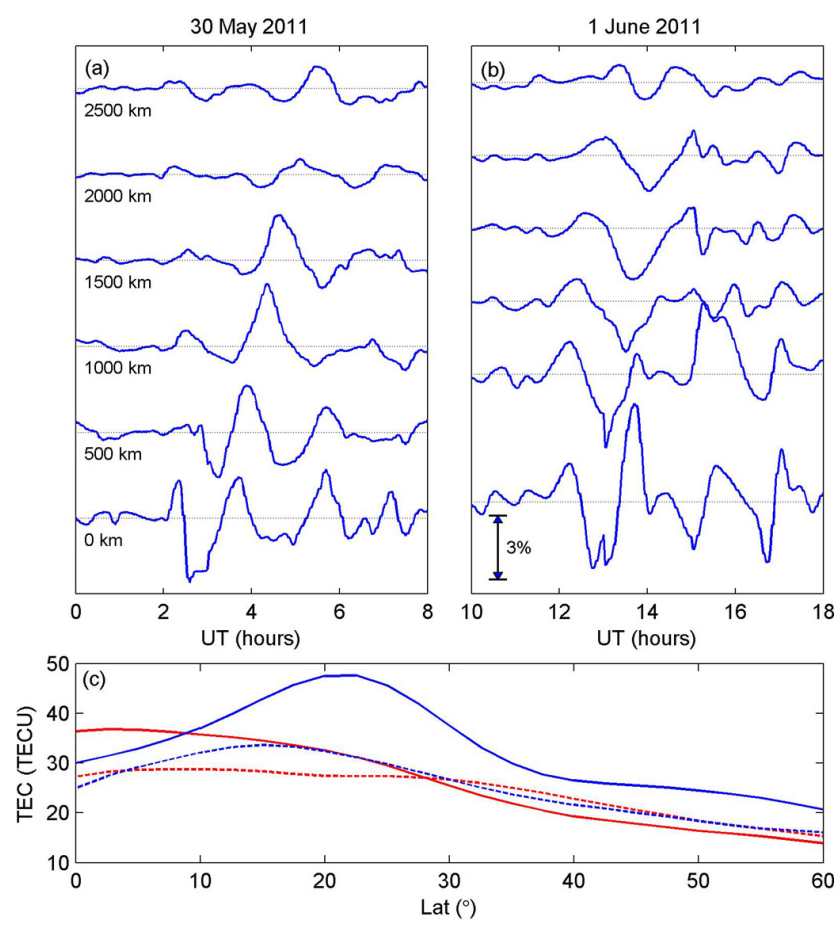

Fig. 6. (a), (b) Temporal variations in relative amplitude at fixed horizontal locations, $x$, ranging from 0 to $2500 \mathrm{~km}$ at intervals of $500 \mathrm{~km}$. (c) Latitudinal variations in background TEC along the $105^{\circ} \mathrm{E}$ meridian during the time of the two events (solid lines) and during quiet time (dashed lines). Solid lines are the latitudinal variation in TEC at 02:00 UT on 30 May (red) and at 12:00 UT on 1 June (blue). The two times correspond to the times of the two LSTID events that occurred in China. Dashed lines represent the corresponding mean values during quiet days on 24-26 May.

$105^{\circ} \mathrm{E}$ meridian during the time of the two events (solid lines). Also shown in the figure are the average variations in TEC during quiet time (dashed lines). Compared with the quiet-time value, there is a strong enhancement in TEC at all latitudes during the nighttime of 1 June. In the latitude range $19-30^{\circ} \mathrm{N}$, where the TIDs were observed, the mean TEC during the nighttime of 1 June exceeded the TEC at noon on 30 May by $43 \%$ (see Fig. 6c). An enhanced electron density can cause strong ion drag. This leads to severe dissipation of LSTIDs, even if they were traveling during the night. Strong nighttime enhancements in TEC are usually observed at low latitudes during both disturbed and quiet times (Unnikrishnan et al., 2002; Jain et al., 2011), and dissipation of LSTIDs due to ion drag has previously been reported by Tsugawa et al. (2004) and Ding et al. (2012).

The LSTID events were observed during the same time intervals by the ionosonde chain in southern China. Figure 7 shows temporal variations in virtual heights at detection frequencies from 6 to $10 \mathrm{MHz}$, in steps of $2 \mathrm{MHz}$. The virtual heights and frequencies were read from the $F$ layer trace in the ionograms recorded by the ionosondes at Wuhan $\left(30.5^{\circ} \mathrm{N}\right)$, Nanning $\left(22.8^{\circ} \mathrm{N}\right)$, Sanya $\left(18.3^{\circ} \mathrm{N}\right)$, 

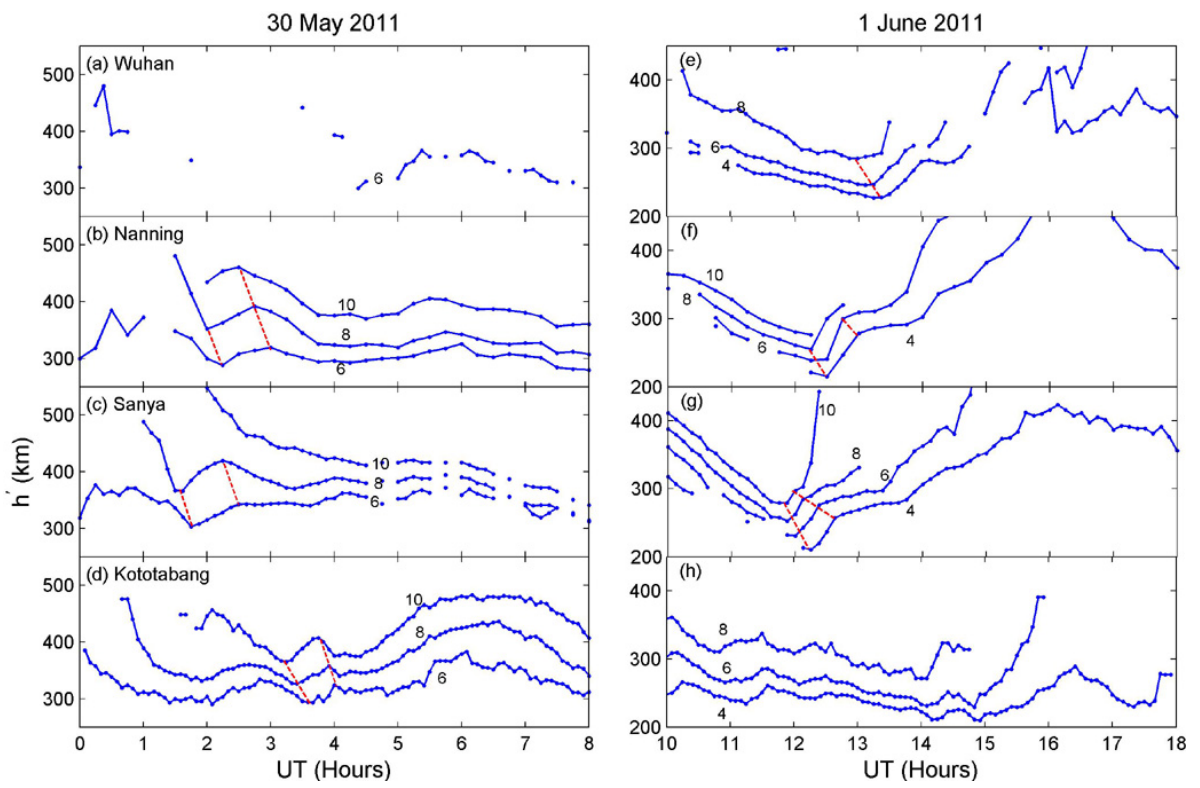

Fig. 7. Temporal variations in virtual height $\left(h^{\prime}\right)$ at detection frequencies ranging from 4 to $10 \mathrm{MHz}$, in steps of $2 \mathrm{MHz}$. The data were recorded by the ionosondes at Wuhan $\left(30.5^{\circ} \mathrm{N}, 114.4^{\circ} \mathrm{E}\right)$, Nanning $\left(22.8^{\circ} \mathrm{N}, 108.3^{\circ} \mathrm{E}\right)$, Sanya $\left(18.3^{\circ} \mathrm{N}, 109.6^{\circ} \mathrm{E}\right)$, and an equatorial station at Kototabang $\left(0.2^{\circ} \mathrm{S}, 100.3^{\circ} \mathrm{E}\right)$. The red dashed lines in each plot connect the peaks and valleys of the variations at different frequencies.

and Kototabang $\left(0.2^{\circ} \mathrm{S}\right)$. Figure 7 shows that small perturbations with amplitudes of 3-4\% occurred during the periods of the two TID events. On 30 May, such a perturbation first occurred at Sanya $\left(18.3^{\circ} \mathrm{N}\right)$ at $01: 30-02: 30 \mathrm{UT}$ (09:30-11:00 LT). It then propagated northward and arrived at Nanning $\left(23.8^{\circ} \mathrm{N}\right)$ approximately 18 min later. The perturbation was not found at Wuhan $\left(30^{\circ} \mathrm{N}\right)$ because of the lack of an $F$ layer trace during that time interval, due to the influence of sporadic $E$ layer occurrence, which blocks highfrequency signals from reaching the $F$ layer. On 1 June, the perturbation can be found in the evening at 11:30-13:30 UT (19:30-21:30 LT). It took $\sim 20 \mathrm{~min}$ for the nighttime LSTID to propagate from Sanya $\left(18.3^{\circ} \mathrm{N}\right)$ to Nanning $\left(23.8^{\circ} \mathrm{N}\right)$, upon which it took $\sim 50 \mathrm{~min}$ to subsequently arrive at Wuhan $\left(30^{\circ} \mathrm{N}\right)$. The time lags of the peaks of the virtual-height series observed at these ionosonde stations coincide with those obtained from the 2-D TEC perturbation maps. This implies that the GPS and ionosondes observed the same LSTID events. Note that, upon inspection of Fig. 7, for both events the peaks and valleys of the virtual height perturbations appear earlier at higher than at lower frequencies, indicating a downward vertical phase velocity. This indicates that the LSTIDs were manifestations of atmospheric gravity waves (AGWs), as previously addressed by many authors (BeckerGuedes et al., 2004; Klausner et al., 2009; Ding et al., 2012). Figure $7 \mathrm{e}-\mathrm{g}$ shows an obvious decline in $F$ layer virtual heights during the early evening (i.e., $\sim 10: 00-12: 00$ UT) followed by an increase. This possibly resulted from the fall of the ionosphere in the early evening due to the loss of solar radiation, and a subsequent rise due to increasing equatorward-blowing thermospheric winds.

If the two LSTID events observed in southern China had propagated northward and passed across Kototabang $\left(0.2^{\circ} \mathrm{S}\right)$ before they reached Sanya $\left(18.3^{\circ} \mathrm{N}\right)$, there would have been some perturbations at Kototabang $\left(0.2^{\circ} \mathrm{S}\right)$ about $1 \mathrm{~h}$ before they were observed at Sanya $\left(18.3^{\circ} \mathrm{N}\right)$. However, such perturbations were not found at Kototabang during the expected time (see Fig. 7d, h). This implies that the northward TIDs originated between Kototabang $\left(0.2^{\circ} \mathrm{S}\right)$ and Sanya $\left(18.3^{\circ} \mathrm{N}\right)$.

\section{Discussion}

LSTIDs play an important role in the dynamic variation of storm-time ionosphere (Shiokawa et al., 2002; Jin et al., 2008). They are frequently observed through GPS measurements or by ionosondes in middle- and high-latitude regions such as North America (Nicolls et al., 2004; Ding et al., 2007, 2008) and Europe (Borries et al., 2009). They are also commonly observed in middle- and low-latitude regions such as Japan (Tsugawa et al., 2004), Brazil (Klausner et al., 2009; de Abreu et al., 2010), and China (Ding et al., 2012). Most observations reveal equatorward-propagating LSTIDs. Our previous study observed two equatorwardtraveling LSTIDs on 28 May during the main phase of the same storm in China (Ding et al., 2012). Comparing the equatorward-propagating TIDs with their poleward-traveling counterparts in the present study, we found that there are three features that distinguish poleward- from equatorwardpropagating TIDs. 
First, compared with equatorward-propagating LSTIDs, poleward-traveling LSTIDs propagate across a smaller latitudinal range. During the same storm, southwestwardpropagating LSTIDs over China were observed across a wide area from the far north to central China, covering a latitudinal range of $29-53^{\circ} \mathrm{N}$ (i.e., magnetic latitudes 18 $42^{\circ} \mathrm{N}$; Ding et al., 2012). LSTIDs of similar scales also occurred in Japan during a similar time interval, indicating that the source regions may be located at high latitudes in the north of Japan; they are thought to be due mostly to auroral activities. Similar latitudinal ranges of equatorwardpropagating LSTIDs over Japan have been reported by Tsugawa et al. (2004), while northward-propagating TIDs were only visible in southern China over the latitudinal range of $\sim$ $18.3-35^{\circ} \mathrm{N}$ (i.e., geomagnetic latitudes of $\sim 7.3-24^{\circ} \mathrm{N}$ ), as evidenced by the TEC perturbation maps. Ionosonde observations showed a narrower latitudinal range of $18.3-23.8^{\circ} \mathrm{N}$ for the first northward-propagating TID and $18.3-30^{\circ} \mathrm{N}$ for the second. This is because the GPS network can detect TIDs over a wider area than ionosondes. The limitation in latitudinal range of the poleward-propagating TIDs indicates that the northward-propagating TIDs in our study are localized rather than a global phenomenon.

Second, poleward-propagating TIDs exhibit much smaller amplitudes than equatorward-propagating TIDs. Based on GPS observations, both poleward- and equatorwardpropagating LSTIDs have absolute amplitudes of 12 TECU, which correspond to relative amplitudes of 2$5 \%$. Although GPS TEC data indicate a similar amplitude range for equatorward- and poleward-propagating TIDs, ionosonde observations exhibit significant differences. As for equatorward-propagating LSTIDs, a maximum amplitude of $\sim 60 \mathrm{~km}$ can be found in the temporal variation of the virtual heights (Ding et al., 2012), corresponding to a relative amplitude of $\sim 20 \%$. Equatorward-propagating LSTIDs with similar amplitudes were observed during stormtime in Brazil (Klausner et al., 2009), while the poleward-propagating TIDs in the present study exhibit an amplitude of $15 \mathrm{~km}(4 \%$; see Fig. 7).

Third, the poleward-traveling LSTIDs propagated at higher elevations than the equatorward-moving TIDs. The elevation of LSTIDs can be estimated by dividing their horizontal by their vertical velocities. The vertical velocity can be calculated using the virtual heights and times of the peaks and valleys of two nearby frequencies (Klausner et al., 2009). Our calculation shows that both polewardpropagating LSTIDs in the present study have an elevation of $\sim 11^{\circ}$, while the equatorward-propagating LSTIDs in Ding et al. (2012) have an elevation of $\sim 8^{\circ}$.

Given these features, it seems that these polewardtraveling LSTIDs did not originate from the auroral region in the Southern Hemisphere. LSTIDs were frequently observed in the Southern Hemisphere through ionosondes and GPS. However, few of them were observed to propagate across the equator and reach Northern Hemisphere (de Abreu et al.,
2010; de Jesus et al., 2010). In our study, the geomagnetic storm was in its recovery phase on 30 May and 1 June 2011, and there is no notable increase in the AE index. A small amplitude and a narrow propagation range imply that these LSTIDs may either have been excited in some source region far away from southern China, and hence propagated for a long distance before arriving there, or they may have been excited by some local source near southern China. As regards the former possibility, dissipation of LSTIDs during their long-distance propagation could have reduced the amplitude and led to a narrow detectable horizontal range. However, this possibility is weakened by the fact that the LSTIDs were not observed by the ionosonde at Kototabang $\left(0.2^{\circ} \mathrm{S}\right)$, where they should have come from. Moreover, if these polewardpropagating LSTIDs were stimulated in the southern auroral region and subsequently propagated across the equator to reach China, the elevation of these LSTIDs should be lower than that of the southward-propagating LSTIDs. This is inconsistent with our estimation, which indicates an elevation of $\sim 8^{\circ}$ for the equatorward-traveling LSTIDs and $\sim 11^{\circ}$ for their poleward-moving counterparts. Therefore, these poleward-propagating LSTIDs cannot have been excited in the southern auroral region, although such auroralexcited, cross-equator-propagating LSTIDs have been observed previously over Japan (Lei et al., 2008).

Taking into account the limited latitudinal range and relatively higher elevation, it is likely that these polewardpropagating LSTIDs were excited by some local source near southern China. Excitation of secondary LSTIDs during the dissipation of some primary medium-scale traveling ionospheric disturbances (MSTIDs) from the lower atmosphere may be one possible mechanism (Vadas and Liu, 2009). Numerical modeling by Vadas and Liu (2009) showed that some disturbances in the lower atmosphere, such as a deep convective plume, could stimulate small- and medium-scale gravity waves. Such medium-scale TIDs are frequently observed at middle and low latitudes (Ding et al., 2011; Valladares and Hei, 2012). These waves propagate obliquely upward into the lower thermosphere and create a body force there through dissipation, which in turn excites large-scale gravity waves. Such types of large-scale secondary waves have been observed as LSTIDs by the TIDDBIT ionospheric sounder (Vadas and Crowley, 2010). The modeling work of Vadas and Liu (2009) predicted a wavelength, horizontal velocity, and amplitude in TEC of such secondary LSTIDs of $\sim 2100 \mathrm{~km}$, $\sim 500 \mathrm{~m} \mathrm{~s}^{-1}$, and up to $8 \%$, respectively. These parameters are very close to our results $\left(\sim 2600 \mathrm{~km}, 410 \mathrm{~m} \mathrm{~s}^{-1}\right.$, and 3$5 \%$, respectively). However, the model results imply global propagation of such kinds of large-scale, secondary TIDs, while global propagation was not observed in our study. The limitation of the latitudinal range is closely related to the strong dissipation due to ion drag at low latitudes, as discussed in the previous section. 


\section{Conclusions}

While most LSTIDs propagate equatorward, polewardpropagating LSTIDs have been reported by several authors (Lei et al., 2008; Bruinsma and Forbes, 2009; Zhou et al., 2012), most of which were observed at middle and low latitudes. In this paper, we used TEC data from 241 GPS receivers in and around China to monitor polewardpropagating LSTIDs at low latitudes during a medium-scale geomagnetic storm from 27 May to 1 June 2011. The GPS observations were combined with observations from four ionosondes, located at Wuhan $\left(30.5^{\circ} \mathrm{N}, 114.4^{\circ} \mathrm{E}\right)$, Nanning $\left(22.8^{\circ} \mathrm{N}, 108.3^{\circ} \mathrm{E}\right)$, Sanya $\left(18.3^{\circ} \mathrm{N}, 109.6^{\circ} \mathrm{E}\right)$, and an equatorial station at Kototabang $\left(0.2^{\circ} \mathrm{S}, 100.3^{\circ} \mathrm{E}\right)$. Our results are summarized as follows.

1. We observed two poleward-propagating LSTIDs in southern China. One occurred on the morning of 30 May 2011, propagating northeastward with amplitude, horizontal velocity, and propagation azimuth (east of north) of -1.4 to $1.0 \mathrm{TECU}, 410 \pm 66 \mathrm{~m} \mathrm{~s}^{-1}$, and $21 \pm 6^{\circ}$, respectively. The other was observed during the evening of 1 June 2011, propagating northwestward with amplitude, horizontal velocity, and azimuth of \pm 2 TECU, $319 \pm 52 \mathrm{~m} \mathrm{~s}^{-1}$, and $333 \pm 9^{\circ}$, respectively.

2. Both poleward-propagating LSTIDs experienced severe dissipation during their propagation from south to north. The amplitude of the morning event on 30 May decreased from $3 \%$ to $1 \%$ after it traveled $2500 \mathrm{~km}$ from the south, while the amplitude of the nighttime event on 1 June decreased from $5 \%$ to $1 \%$. Although the initial amplitude of the nighttime LSTID was $\sim 60 \%$ larger than that of the morning event, the nighttime event dissipated more quickly than the morning event, possibly because of the strong nighttime enhancement in background TEC during stormtime, which leads to strong ion-drag dissipation in the evening.

3. The poleward-propagating LSTIDs in southern China exhibit a narrower latitudinal range (geomagnetic latitude $\left.\sim 7.3-24^{\circ} \mathrm{N}\right)$, a smaller amplitude, and a slightly higher elevation compared with equatorwardpropagating LSTIDs observed in the same region. Given these features, the poleward-traveling LSTIDs were likely excited by some local source near southern China. Excitation of secondary LSTIDs during the dissipation of some primary MSTIDs from the lower atmosphere could be a possible mechanism (Vadas and Liu, 2009).

Acknowledgements. We acknowledge the IGS for providing GPS network data via the Internet. The data of Global Ionospheric Maps used in this study were acquired as part of NASA's Earth Science Data Systems and archived and distributed by the Crustal Dynamics Data Information System (CDDIS). The authors are thank- ful to the World Data Center (WDC) for Ionosphere, Tokyo, National Institute of Information and Communications Technology for providing ionogram data in the Internet. We acknowledge the use of data from the Chinese Meridian Project. This work was supported by the National Natural Science Foundation of China (grants 41274162, 40974089 and 41131066), the Chinese Academy of Sciences (KGCXZ-EW-407-2, KZZD-EW-01-2), and the National Key Basic Research Program of China (2011CB811405).

Topical Editor K. Kauristie thanks S. Jin and P. R. Fagundes for their help in evaluating this paper.

\section{References}

Becker-Guedes, F., Sahai, Y., Fagundes, P. R., Lima, W. L. C., Pillat, V. G., Abalde, J. R., and Bittencourt, J. A.: Geomagnetic storm and equatorial spread-F, Ann. Geophys., 22, 3231-3239, doi:10.5194/angeo-22-3231-2004, 2004.

Borries, C., Jakowski, N., and Wilken, V.: Storm induced large scale TIDs observed in GPS derived TEC, Ann. Geophys., 27, 16051612, doi:10.5194/angeo-27-1605-2009, 2009.

Bruinsma, S. L. and Forbes, J. M.: Properties of traveling atmospheric disturbances (TADs) inferred from CHAMP accelerometer observations, Adv. Space Res., 43, 369-376, 2009.

de Abreu, A. J., Fagundes, P. R., Sahai, Y., de Jesus, R., Bittencourt, J. A., Brunini, C., Gende, M., Pillat, V. G., Lima, W. L. C., Abalde, J. R., and Pimenta, A. A.: Hemispheric asymmetries in the ionospheric response observed in the American sector during an intense geomagnetic storm, J. Geophys. Res., 115, A12312, doi:10.1029/2010JA015661, 2010.

de Jesus, R., Sahai, Y., Guarnieri, F. L., Fagundes, P. R., de Abreu, A. J., Fecker-Guedes, F., Brunini, C., Gende, M., Cintra, T. M. F., de Souza, V. A., Pillat, V. G., and Lima, W. L. C.: Effects observed in the ionospheric F-region in the South American sector during the intense geomagnetic storm of 14 December 2006, Adv. Space Res., 46, 909-920, 2010.

Ding, F., Wan, W., Ning, B., and Wang, M.: Large scale traveling ionospheric disturbances observed by GPS TEC during the magnetic storm of October 29-30, 2003, J. Geophys. Res., 112, A06309, doi:10.1029/2006JA012013, 2007.

Ding, F., Wan, W., Liu, L., Afraimovich, E. L., Voeykov, S. V., and Perevalova, N. P.: A statistical study of large-scale traveling ionospheric disturbances observed by GPS TEC during major magnetic storms over the years 2003-2005, J. Geophys. Res., 113, A00A01, doi:10.1029/2008JA013037, 2008.

Ding, F., Wan, W., Xu, G., Yu, T., Yang, G., and Wang, J.: Climatology of medium-scale traveling ionospheric disturbances observed by a GPS network in central China, J. Geophys. Res., 116 , A09327, doi:10.1029/2011JA016545, 2011.

Ding, F., Wan, W., Ning, B., Zhao, B., Li, Q., Zhang, R., Xiong, B., and Song, Q.: Two-dimensional imaging of large-scale traveling ionospheric disturbances over China based on GPS data, J. Geophys. Res., 117, A08318, doi:10.1029/2012JA017546, 2012.

Hocke, K. and Schlegel, K.: A review of atmospheric gravity waves and travelling ionospheric disturbances: 1982-1995, Ann. Geophys., 14, 917-940, doi:10.1007/s00585-996-0917-6, 1996.

Hunsucker, R. D.: Atmospheric gravity waves generated in the high-latitude ionosphere: A review, Rev. Geophys., 20, 293-315, doi:10.1029/RG020i002p00293, 1982. 
Jain, A., Tiwari, S., Jain, S., and Gwal, A. K.: Nighttime enhancements in TEC near the crest of northern equatorial ionization anomaly during low solar activity period, Indian Journal of Physics, 85, 1367-1380, doi:10.1007/s12648-011-0159-7, 2011.

Jin, S. G., Luo, O., and Park, P.: GPS observations of the ionospheric F2-layer behavior during the 20th November 2003 geomagnetic storm over South Korea, J. Geod., 82, 883-892, doi:10.1007/s00190-008-0217-x, 2008.

Klausner, V., Fagundes, P. R., Sahai, Y., Wrasse, C. M., Pillat, V. G., and Becker-Guedes, F.: Observations of GW/TID oscillations in the F2 layer at low latitude during high and low solar activity, geomagnetic quiet and disturbed periods, J. Geophys. Res., 114, A02313, doi:10.1029/2008JA013448, 2009.

Lei, J., Burns, A. G., Tsugawa, T., Wang, W., Solomon, S. C., and Wiltberger, M.: Observations and simulations of quasiperiodic ionospheric oscillations and large-scale traveling ionospheric disturbances during the December 2006 geomagnetic storm, J. Geophys. Res., 113, A06310, doi:10.1029/2008JA013090, 2008.

Nicolls, M. J., Kelley, M. C., Coster, A. J., Gonzalez, S. A., and Makela, J. J.: Imaging the structure of a large-scale TID using ISR and TEC data, Geophys. Res. Lett., 31, L09812, doi:10.1029/2004GL019797, 2004.

Shiokawa, K., Otsuka, Y., Ogawa, T., Balan, N., Igarashi, K., Ridley, A. J., Knipp, D. J., Saito, A., and Yumoto, K.: A large-scale traveling ionospheric disturbance during the magnetic storm of 15 September 1999, J. Geophys. Res., 107, 1088, doi:10.1029/2001JA000245, 2002.

Song, Q., Ding, F., Wan, W., Ning, B., and Liu, L.: Global propagation features of large-scale traveling ionospheric disturbances during the magnetic storm of $7 \sim 10$ November 2004, Ann. Geophys., 30, 683-694, doi:10.5194/angeo-30-683-2012, 2012.
Tsugawa, T., Saito, A., and Otsuka, Y.: A statistical study of large-scale traveling ionospheric disturbances using the GPS network in Japan, J. Geophys. Res., 109, A06302, doi:10.1029/2003JA010302, 2004.

Unnikrishnan, K., Balachandran Nair, R., and Venugopal, C.: A comparative study of night-time enhancement of TEC at a low latitude station on storm and quiet nights including the local time, seasonal and solar activity dependence, Ann. Geophys., 20, 1843-1850, doi:10.5194/angeo-20-1843-2002, 2002.

Vadas, S. L. and Crowley, G.: Sources of the traveling ionospheric disturbances observed by the ionospheric TIDDBIT sounder near Wallops Island on 30 October 2007, J. Geophys. Res., 115, A07324, doi:10.1029/2009JA015053, 2010.

Vadas, S. L. and Liu, H.: Generation of large-scale gravity waves and neutral winds in the thermosphere from the dissipation of convectively generated gravity waves, J. Geophys. Res., 114, A10310, doi:10.1029/2009JA014108, 2009.

Valladares, C. E. and Hei, M. A.: Measurement of the Characteristics of TIDs Using Small and Regional Networks of GPS Receivers during the Campaign of 17-30 July of 2008, Int. J. Geophysics, 2012, A 548784, doi:10.1155/2012/548784, 2012.

Zhou, C., Zhao, Z., Yang, G., Chen, G., Hu, Y., and Zhang, Y.: Evidence of low-latitude daytime large-scale traveling ionospheric disturbances observed by high-frequency multistatic backscatter sounding system during a geomagnetically quiet period, J. Geophys. Res., 117, A06302, doi:10.1029/2012JA017605, 2012. 